\title{
In this issue
}

Duangruthai Sridaeng, Wannisa Jitaree, Preecha Thiampanya and Nuanphun Chantarasiri

Preparation of rigid polyurethane foams using low-emission catalysts derived from metal acetates and ethanolamine

DOI 10.1515/epoly-2016-0021

e-Polymers 2016; 16(4): 265-275
Full length article: Metal acetateethanolamine complexes $\left[\mathrm{M}(\mathrm{OAc})_{2}(\mathrm{EA}), \mathrm{M}=\mathrm{Cu}\right.$ and $\left.\mathrm{Zn}\right]$ were synthesized in one step and used as low-emission catalysts in the preparation of rigid polyurethane (RPUR) foams.

Keywords: copper-ethanolamine complex; low-emission catalyst; metal acetate; rigid polyurethane foam; zincethanolamine complex.
Stefan Oprea and Veronica Oprea Biodegradation of crosslinked polyurethane acrylates/guar gum composites under natural soil burial conditions

DOI 10.1515/epoly-2016-0038 e-Polymers 2016; 16(4): 277-286
Full length article: This paper describes soil burial biodegradation processes of the high crosslinked polyurethane acrylates and their composites with different content of the guar gum. High biodegradability of these materials, although are highly crosslinked, is provide by the modified soybean oil and guar gum from their structures. This kind of material is important for the natural environmental.

Keywords: biodegradable composites; guar gum; mechanical properties; polyurethane acrylates; soil burial.
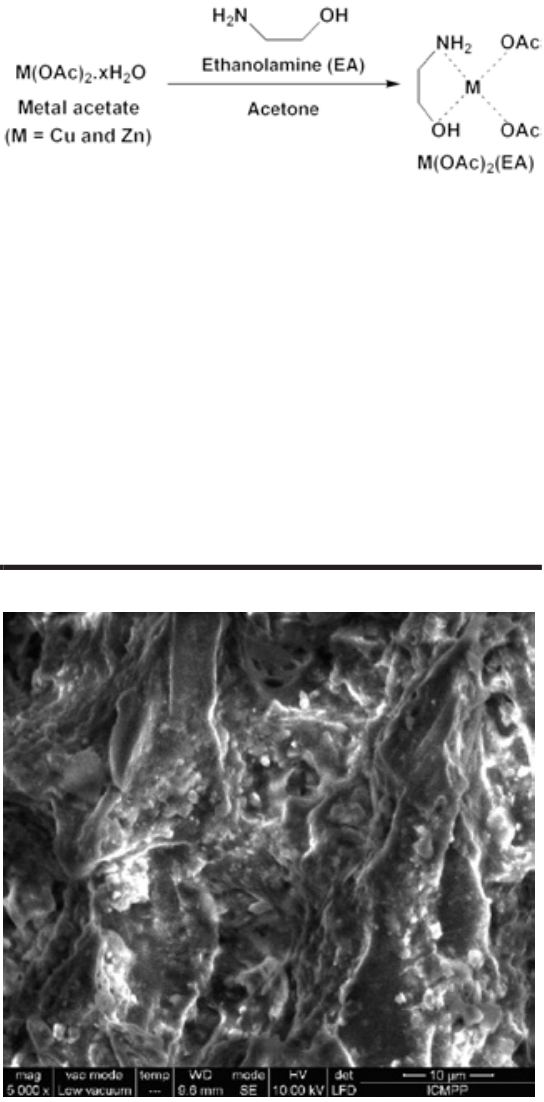
Szymon Żaczek, Agnieszka

Pająk, Rafat Anyszka and Grażyna

Janowska

Influence of phthalocyanine pigments on the properties of

flame-retardant elastomeric composites based on styrenebutadiene or acrylonitrilebutadiene rubbers

DOI 10.1515/epoly-2016-0046

e-Polymers 2016; 16(4): 287-294
Full length article: A study about the influence of the impartment of phthalocyanine pigments into elastomeric composites was conducted. Acrylonitrile-butadiene (NBR) and styrene-butadiene (SBR) rubbers crosslinked by either sulfur or dicumyl peroxide were filled with non-toxic flame retardants $\left(\mathrm{Mg}(\mathrm{OH})_{2}\right.$ or $\left.\mathrm{Al}(\mathrm{OH})_{3}\right)$ and thermally stable pigments (zinc or chloroaluminum phthalocyanines). Determination of cross-linking degree, mechanical properties, aging coefficient, thermal analysis (TG, DTG, DTA) and flammability tests (oxygen index, combustion in air) were carried out.

Keywords: acrylonitrile-butadiene rubber; elastomeric composites; flammability; phthalocyanine pigments; styrene butadiene rubber.
Yonglin Lei, Yuanjie Shu, Jinhua Peng, Yongjian Tang and Jichuan Huo

Synthesis and properties of low coefficient of thermal expansion copolyimides derived from biphenyltetracarboxylic dianhydride with p-phenylenediamine and 4,4'-oxydialinine

DOI 10.1515/epoly-2015-0267 e-Polymers 2016; 16(4): 295-302
Full length article: Three copolyimides from s-BPDA, a-BPDA, 4,4'-ODA, PDA exhibited high $\mathrm{T}_{\mathrm{g}}$, high thermal stability, great ultimate strength and low CTE. The copolymerization of s-BPDA/PDA with a small number of 4, 4'-ODA/a-BPDA was a useful means for enhancing flexibility without sacrificing low CTE.

Keywords: 4,4'-oxydialinine;

biphenyltetracarboxylic

dianhydride; copolyimide; low-CTE;

p-phenylenediamine.
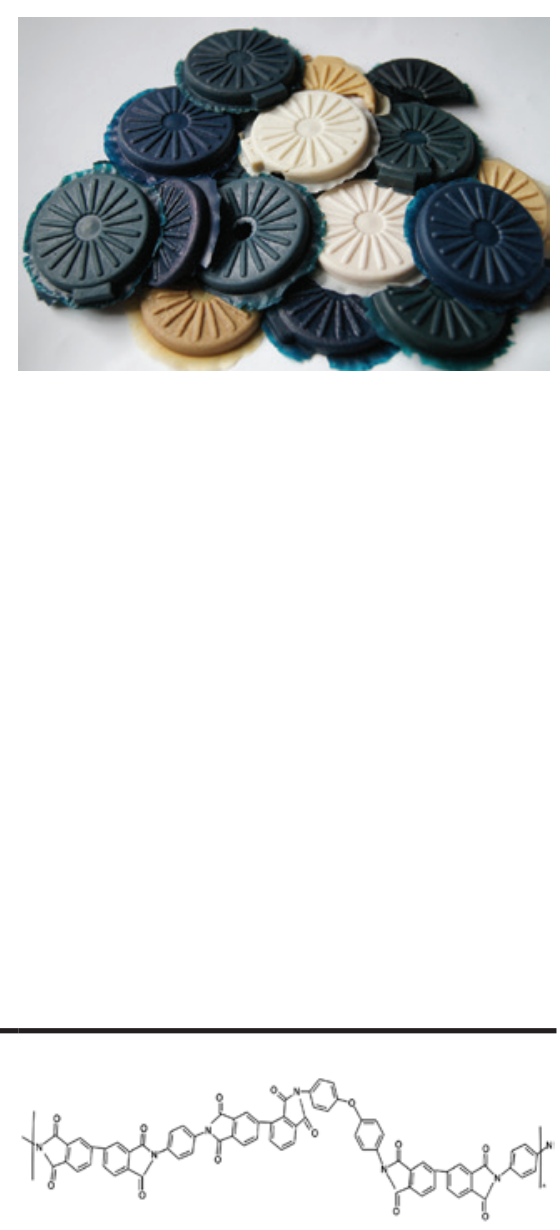
Yan-Hua Cai and Li-Sha Zhao

Thermal behavior of modified

poly(L-lactic acid): effect of aromatic multiamide derivative based on $1 \mathrm{H}$-benzotriazole

DOI 10.1515/epoly-2016-0052 e-Polymers 2016; 16(4): 303-311
Full length article: The nonisothermal crystallization behavior of PLLA is related to the set final melting temperature as well as $\mathrm{PB}$ concentration. When the set final melting temperature is $190^{\circ} \mathrm{C}$, PLLA/ PB samples exhibit the highest $T_{c p}$, indicating $190^{\circ} \mathrm{C}$ is the optimization blending temperature for PLLA/PB. In addition, under the same final melting temperature, PLLA $/ 2 \%$ PB has the highest $T_{c p}$, further confirming the best effect of crystallization at $2 \mathrm{wt} \%$ PB. In particular, when the set final melting temperature is $210^{\circ} \mathrm{C}$, the $T_{c p}$ between PLLA/2\%PB and PLLA/0.5\%PB appears the maximum difference $5.2^{\circ} \mathrm{C}$.

Keywords: aromatic multiamide; crystallization; nucleating agent; poly(L-lactic acid); thermal behavior.
Dun Chen, Tunsagnl Awut, Bin Liu, Yali Ma, Tao Wang and Ismayil Nurulla Functionalized magnetic $\mathrm{Fe}_{3} \mathrm{O}_{4}$ nanoparticles for removal of heavy metal ions from aqueous solutions

DOI 10.1515/epoly-2016-0043 e-Polymers 2016; 16(4): 313-322
Full length article: $\mathrm{Fe}_{3} \mathrm{O}_{4}$ nanoparticles (MNP) were coated with 3-aminopropyltriethoxy-silane (APTES), resulting in anchoring of primary amine groups on the surface of the particles, then four kinds of novel magnetic adsorbents $\left(\mathrm{Fe}_{3} \mathrm{O}_{4} @\right.$ $\mathrm{SiO}_{2}-\mathrm{NH}$-HCGs) were formed by grafting of different heterocyclic groups (HCG) on amino groups via substitution reaction.

Keywords: adsorbent; $\mathrm{Fe}_{3} \mathrm{O}_{4}$ nanoparticles; heavy metal ions removal; magnetic separation; removal efficiency.
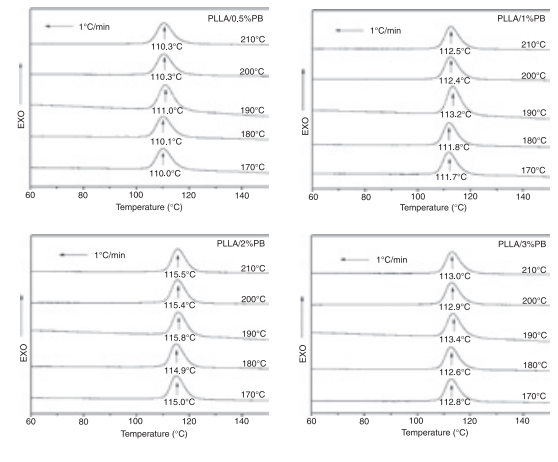$$
\sqrt{1 \% \times P B}
$$

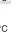$$
{ }_{180^{\circ} \mathrm{C}}
$$

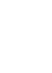


J. Sahari and M.A. Maleque

Effect of oil palm ash on the mechanical and thermal properties of unsaturated polyester composites

DOI 10.1515/epoly-2016-0079

e-Polymers 2016; 16(4): 323-329
Full length article: This research was carried out to evaluate the effect of oil palm ash (OPA) on the mechanical and thermal properties of biocomposites. Different compositions of OPA were introduced to unsaturated polyester by using a simple casting method with methyl ethyl ketone peroxide as a hardener. The specimens were prepared based on ASTM D5083 standards for tensile testing.

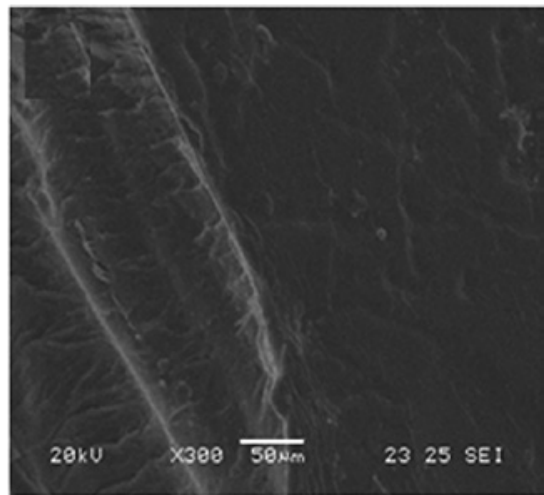

Keywords: biocomposites; mechanical; oil palm ash (OPA); thermal; unsaturated polyester (UPE).

Full length article: $\mathrm{BC}$ production from Gluconacetobacter xylinus MTCC 7795 in HS media modified with various carbon sources has been investigated in shake flask culture. Growth kinetics and Physiochemical properties of BC produced was studied using FTIR and XRD.

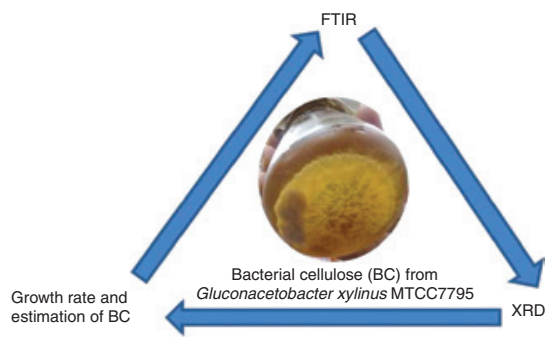

Keywords: bacterial cellulose; biopolymer; FTIR; XRD.
Xin Sanfa and Wang Xinhou Investigation into the effect of the angle of dual slots on an air flow field in melt blowing via numerical simulation

DOI 10.1515/epoly-2016-0057 e-Polymers 2016; 16(4): 337-342
Full length article: Structure and properties of Polytetrafluoroethylene (PTFE) fibers were characterized thoroughly. It was found that the longitudinal surface of the fiber was not smooth and full of grooves. The cross sections of fibers were sheet-like and irregular.

Keywords: air flow field; angle of dual slots; melt blowing; numerical simulation; polymer.

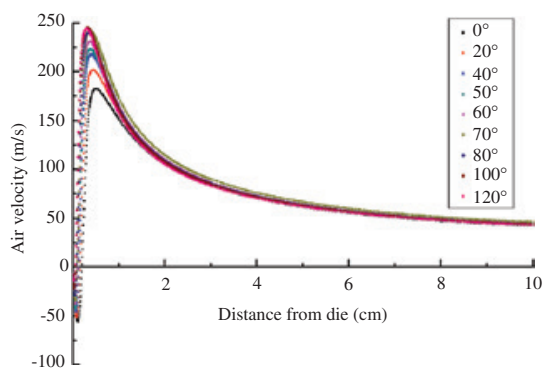


Ya-Juan Su, Ze-Xin Ma and Jian-Hua Huang

Simulation study on the assembly of rod-coil diblock copolymers within coil-selective nanoslits

DOI 10.1515/epoly-2015-0275 e-Polymers 2016; 16(4): 343-349
Full length article: Dissipative particle dynamics simulations are performed to study the self-assembly of rod-coil (RC) diblock copolymers confined in a slit with two coil-selective surfaces. The effect of rod length and slit thickness on the assembly structure is investigated. A morphological phase diagram as a function of slit thickness

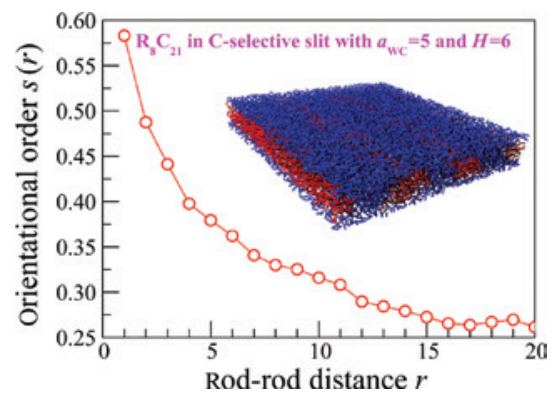
and rod length is presented.

Keywords: confinement; dissipative particle dynamics; rod-coil diblock copolymer; rod orientation; selfassembly structure. 